\title{
Improving Mass Accuracy of High Performance Liquid Chromatography/ Electrospray Ionization Time-of-Flight Mass Spectrometry of Intact Antibodies
}

\author{
Himanshu S. Gadgil, Gary D. Pipes, Thomas M. Dillon, \\ Michael J. Treuheit, and Pavel V. Bondarenko \\ Department of Pharmaceutics, Amgen, Thousand Oaks, California, USA
}

\begin{abstract}
The glycosylation profile of intact antibody due to the galactose and fucose heterogeneity in the N-linked sugars was determined with instrument resolution of 5000 and 10,000. After deconvolution of electrospray ionization mass spectra to complete convergence, several extra peaks appeared in addition to the peaks observed in the original mass spectra. The artificial peaks were avoided if deconvolution was stopped after a smaller number of iterations. A standard antibody was used as an external calibrant to minimize mass measurement errors during long-period experiments. Precision of four consecutive LC/MS measurements of the same antibody was $10 \mathrm{ppm}( \pm 1.5 \mathrm{Da})$. By using this approach, the masses of 11 intact antibodies were measured. All antibodies containing $\mathrm{N}$-terminal glutamines had a negative mass shift due to the formation of pyroglutamate $(-17 \mathrm{Da})$. Although the pyroglutamate variant of intact antibody was not resolved from the unmodified variant, this modification led to a mass shift proportional to the percentage of N-terminal pyroglutamate. By accurately measuring the mass shift we were able to quantify the abundance of pyroglutamic acid on intact antibodies. Mass accuracy in measuring different antibodies was below $30 \mathrm{ppm}( \pm 4 \mathrm{Da})$. The accurate mass measurement can be an effective tool for monitoring chemical degradations in therapeutic antibodies. (J Am Soc Mass Spectrom 2006, 17, 867-872) (C) 2006 American Society for Mass Spectrometry
\end{abstract}

I gG antibodies are heterodimers with molecular weight of approximately $150 \mathrm{kDa}$ and are composed of two identical heavy chains and two identical light chains linked together through disulfide linkages [1]. In addition to the protein component, IgG molecules contain an N-linked biantennary sugar in the conserved region on each heavy chain [2, 3]. The common post-translational modifications that occur in vivo during production of mammalian recombinant antibodies include C-terminal lysine variants, glycosylation variants, and the formation of $\mathrm{N}$-terminal pyroglutamic acid. The C-terminal lysine residues are often removed by the enzyme carboxypeptidase [4]. The earlier studies of several proteins including antibodies, suggested that N-terminal pyroglutamate $(\mathrm{pE})$ is most likely formed either late in protein translation by cyclization of $\mathrm{N}$-terminal glutamine $(Q)$ or as a post-translational event just before cellular secretion of protein from the cell $[5,6]$.

Published online April 21, 2006

Address reprint requests to Dr. P. V. Bondarenko, Department of Pharmaceutics, Amgen, One Amgen Center Drive, Thousand Oaks, CA 91320-1799, USA. E-mail: pavel.bondarenko@amgen.com
In a recent study [7], a set of almost 300 secreted recombinant human proteins was analyzed by Edman analysis to examine frequency of amino acid at the N-terminus. Glutamine was identified as the most preferred $\mathrm{N}$-terminal residue. It was found at the first position in $10.7 \%$ of the proteins. The authors also suggested that the frequently observed cyclization of $\mathrm{N}$-terminal $\mathrm{Q}$ to $\mathrm{pE}$ provides protection for the secreted proteins against degradation by extracellular aminopeptidase [7]. Out of more than 200 human and murine immunoglobulin heavy and light chains subjected to amino terminal sequence analysis [8], Q residues are largely converted to $\mathrm{pE}$ residues with few exceptions [9]. The glutamic to pyroglutamic acid conversion $[5,10]$ seems to be less often and, if happens, takes place with slower rate than $\mathrm{Q}$ to $\mathrm{pE}$. $\mathrm{N}$-terminal $\mathrm{Q}$ can be converted to $\mathrm{pE}$ during fermentation process and during storage in pharmaceutics with exposure to elevated temperatures and near neutral $\mathrm{pH}[10,11]$. The cyclization was also detected during peptide mapping, which is typically conducted at $\mathrm{pH} 7.5$ and $37^{\circ} \mathrm{C}$, the conditions which facilitate formation of $\mathrm{pE}$ on $\mathrm{N}$-termini of unfolded peptides [12]. 
The LC/MS analysis of IgG molecules is challenging due to their high molecular mass, hydrophobic nature, and presence of sugar moieties. Conversely, LC/MS analysis of intact protein is also advantageous because it reduces dramatically the time for sample preparation and data interpretation, compare with peptide mapping, and sequencing. Furthermore it also minimizes the chance of introducing putative modifications, which are often observed during peptide mapping. In this paper, we discuss methods to improve mass accuracy of IgG molecules and the utility of the improved mass measurement for quantification of N-terminal pyroglutamate.

\section{Materials and Methods}

\section{Materials}

Eleven recombinant monoclonal IgG antibodies used in this study were produced and purified at Amgen. $\mathrm{N}$-terminal residues of heavy chains of seven antibodies were glutamines, which are susceptible to conversion to pyroglutamate. N-terminal residues of heavy chains of four other antibodies and light chains of all antibodies were either glutamic or aspartic acid residues, which are stable with respect to cyclization. Trifluoroacetic acid (TFA) was obtained from Pierce, Rockford, IL. $\mathrm{N}$-propanol (NPA) and water were obtained from Burdick and Jackson, Muskegon, MI, no. 322-1.

\section{Reversed-Phase Chromatography}

The reversed-phase HPLC method was adopted from reference[13] with minor modifications. Reversedphase separation was carried out using an Agilent 1100 series CapLC equipped with an Agilent Zorbax SB 300 C8 $50 \times 1 \mathrm{~mm}$ column. A $\mu \mathrm{g}$ protein sample was typically injected on the column. The column was initially equilibrated with $90 \%$ Solvent A $(0.1 \%$ aqueous TFA) and $10 \%$ Solvent B (90\% NPA and $0.1 \%$ aqueous TFA) for 2 min followed by a 1 min step gradient from 5 to $21 \%$ B. Elution was achieved with a liner gradient of 21 to $27 \%$ B over $20 \mathrm{~min}$. The flow rate and temperature were maintained at $50 \mu \mathrm{l} / \mathrm{min}$ and $75^{\circ} \mathrm{C}$ throughout the run.

\section{ESI-TOF Mass Spectrometry}

Mass spectrometric analysis was carried out in a positive ion mode on a Waters LCT Premier and Waters Q-TOF Micro orthogonal TOF instruments equipped with an ESI source. The analyses on the LCT Premier were carried out either in $\mathrm{V}$ or $\mathrm{W}$ mode with instrument resolution at 5000 and 10,000, respectively. The instruments were calibrated in the $\mathrm{m} / \mathrm{z}$ range of 2500 to 4000 using multiply charged ions of one of the studied antibodies (IgG001) with a calculated MW value of 148,251.2 Da. ESI mass spectra were deconvoluted us-

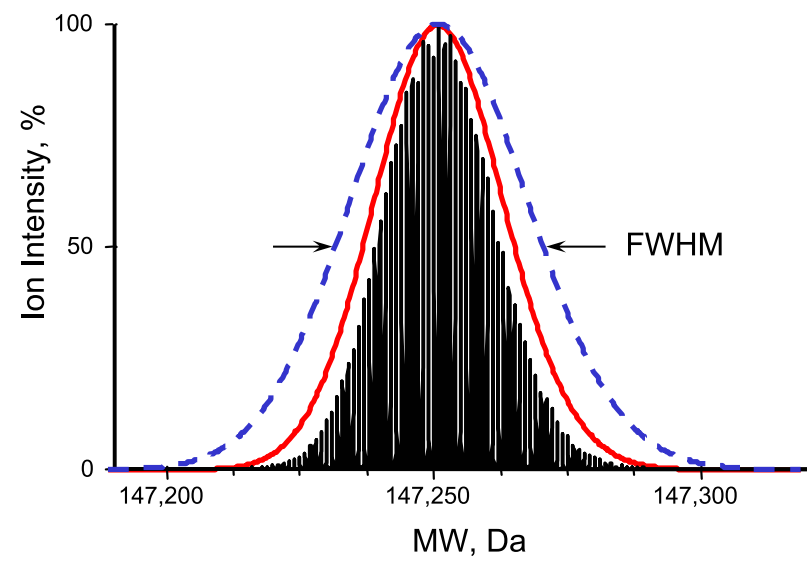

Figure 1. Calculated peak profile for the intact antibody, IgG001, which has an elemental composition of C6516 H10034 N1706 O2082 S52 and a calculated average molecular weight of 147251.2 $\mathrm{Da}$. Calculations were performed with IsoPro software using the following parameters: $1 \times 10^{6}$ molecules; empirical type; all other were default parameters. The calculated peak profiles from narrow to wide are shown as follows: natural isotopic distribution (vertical peaks), profile for instrument resolution of 10,000 (solid red contour line), and 5000 (dashed blue contour line).

ing Waters MassLynx MaxEnt 1 software to obtain the deconvoluted mass.

\section{Results and Discussion}

Figure 1 shows the calculated natural isotopic distribution and calculated peak profiles for the intact antibody IgG001, with instrument resolution set at 5000 and 10,000. The calculations were performed using the IsoPro software (Senko, M.W., http://members.aol.com/ msmssoft/REGISTER.HTM). For IgG001 antibody, the natural isotopic distribution is $22 \mathrm{Da}$ at the full width at half maximum (FWHM). The calculated FMHW is broadened, due to the limited resolving power of a mass spectrometer, to $30 \mathrm{Da}$ for resolution of 10,000 and to $40 \mathrm{Da}$ for resolution of 5000. Therefore, modifications such as $\mathrm{N}$-terminal pyroglutamic acid formation $(-17$ Da) cannot be resolved on the intact IgG molecule. However, these modifications should affect the peak shape of the deconvoluted mass spectrum and shift the measured mass of intact antibody.

The charge distribution profiles of antibody IgG001 in $\mathrm{V}$ and $\mathrm{W}$ modes are shown in Figure 2a. The various peaks observed in the mass $(\mathrm{m} / \mathrm{z})$ spectra in Figure 2a represent successive charge states of the molecular ions of IgG001. These peaks were used to calibrate the mass $(\mathrm{m} / \mathrm{z})$ scale of orthogonal TOF mass spectrometers used in this study. We found that the best mass accuracy for intact antibodies was achieved when the instruments were calibrated with an intact antibody and in the same $\mathrm{m} / \mathrm{z}$ range. The total number of protein ions reaching the detector was approximately two times lower in the $\mathrm{W}$ mode compared with the $\mathrm{V}$ mode, which was anticipated because of the additional ion scattering in the flight tube in $\mathrm{W}$ mode. It was observed that peaks with lower charge state (z) 

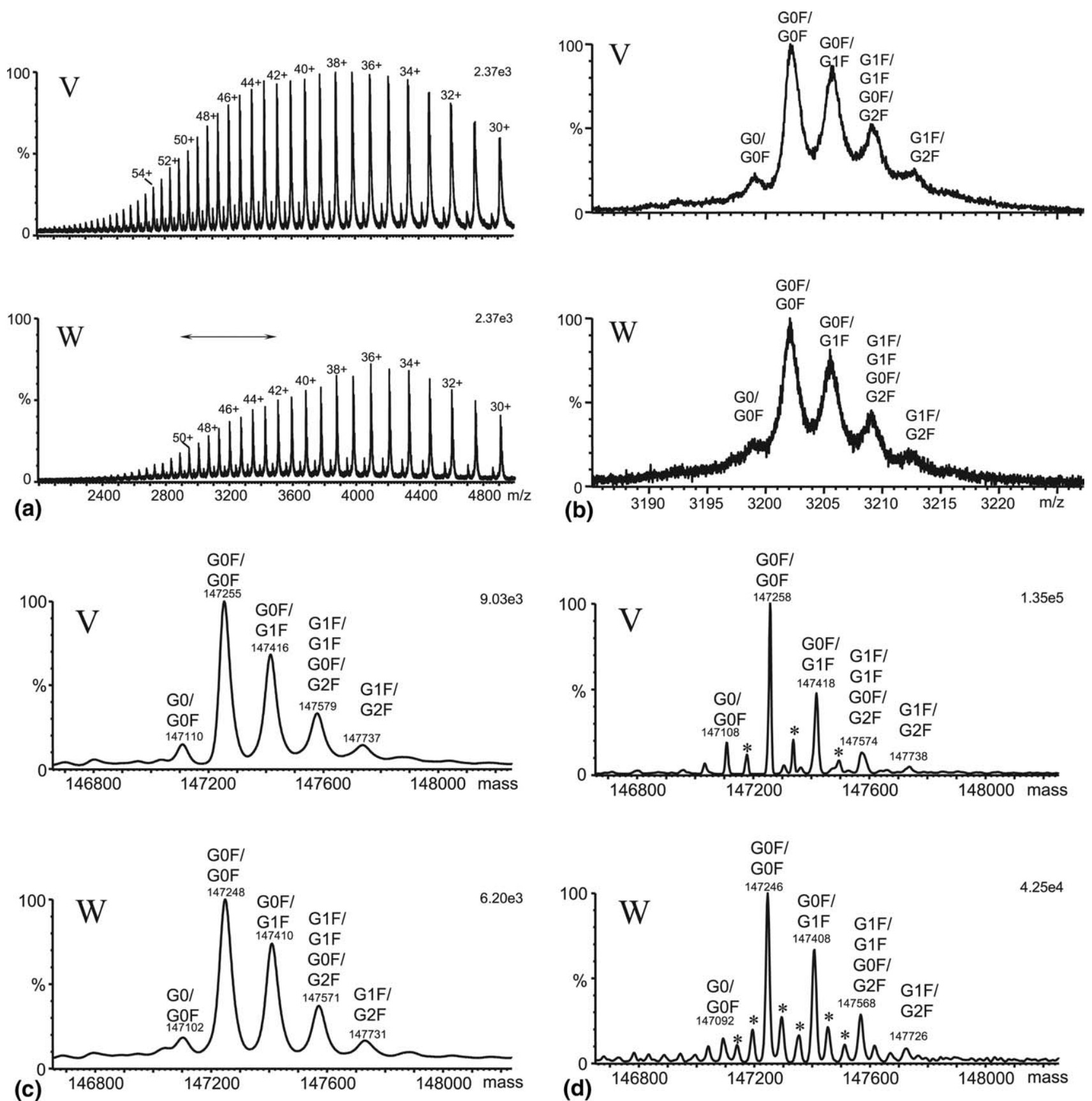

Figure 2. (a) Positive ion ESI mass spectra of an intact monoclonal antibody IgG001, acquired in V mode with resolution 5000 and $\mathrm{W}$ mode with resolution 10,000. The deconvolution range is marked as a double-headed arrow. Ion intensity is in the right top corner. (b) Positive ion ESI mass spectra for 46+ charged ion of an intact monoclonal antibody IgG001 acquired in V mode and W mode. Peak labels represent various glycoforms described in detail in Table 1. (c) Deconvoluted ESI mass spectra of an intact monoclonal antibody IgG001 acquired using V and W modes containing multiple peaks due to the sugar heterogeneity. The following MaxEnt parameters were used for data processing: mass range, 140,000 to $160,000 \mathrm{Da}$; minimum intensity ratio left and right, 50\%; width at half height for uniform Gaussian model, 1.0; number of iterations, 8. Peak labels represent various glycoforms (see Table 1). (d) Deconvoluted ESI mass spectra of an intact monoclonal antibody IgG001 acquired using V and W modes. Deconvolution of the multiply changed ions was performed to complete convergence ( $\sim 40$ iterations). Peaks labeled with an asterisk $\left(^{*}\right)$ are artifacts of deconvolution. For deconvolution, width at half height for uniform Gaussian model was 1.4 for $\mathrm{V}$ mode and 1.2 for $\mathrm{W}$ mode. The different width values were chosen to better illustrate shift of the artificial peaks. All other deconvolution parameters were the same as in (c). 
Table 1. Structures of N-linked sugars commonly found in IgG molecules. Each antibody typically has two glycans, one on each heavy chain

\begin{tabular}{|c|c|c|}
\hline Code & Oligosaccharide structure & $\begin{array}{l}\text { Average } \\
\text { mass }\end{array}$ \\
\hline $\mathrm{G} 2 \mathrm{~F}$ & $\begin{array}{l}\mathrm{G}-\mathrm{GN}-\mathrm{M} \backslash \mathrm{M}-\mathrm{GN}-\mathrm{GN}- \\
\mathrm{G}-\mathrm{GN}-\mathrm{M}^{\prime}\end{array}$ & 1769.6 \\
\hline G1F & 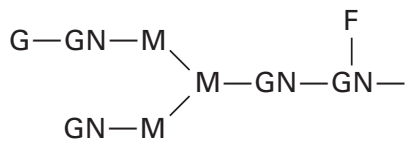 & 1607.5 \\
\hline $\mathrm{GOF}$ & ${ }_{\mathrm{GN}-\mathrm{M}^{\prime}}^{\mathrm{GN}-\mathrm{M}}+\mathrm{MN}-\mathrm{GN}-$ & 1445.4 \\
\hline G0 & ${ }_{\mathrm{GN}}^{\mathrm{GN}-\mathrm{M}} / \mathrm{M}-\mathrm{GN}-\mathrm{GN}-$ & 1299.3 \\
\hline $\mathrm{F}$ & Fucose & 146.1430 \\
\hline G & Glucose & 162.1424 \\
\hline M & Mannose & 162.1424 \\
\hline GN & $\mathrm{N}$-acetylglucosamine & 203.1950 \\
\hline
\end{tabular}

Codes are adapted from reference [3].

exhibit tailing, presumably due to solvent adducts of TFA (+114 Da) and mass resolution of these peaks was lower compare to the peaks with higher z. It was also observed that the tailing was more pronounced in $\mathrm{V}$ mode (Figure $2 \mathrm{a}$ ). Hence, higher $\mathrm{z}$ (lower $\mathrm{m} / \mathrm{z}$ ) peaks were used for deconvolution. The minor satellite peaks in Figure 2a with slightly lower mass than the major peaks are due to the antibody molecules with only one sugar moiety. This was a unique modification of this lot of IgG001, not typical for other antibodies.

The ESI mass spectra acquired in $\mathrm{V}$ and $\mathrm{W}$ modes near a molecular ion with charge $46+$ in the middle of the deconvolution range are shown in Figure $2 \mathrm{~b}$. The sugar profile is typical for antibodies and generated by the loss of terminal galactose (162 Da) and fucose (146 Da) residues (Table 1) similar to profiles in reference [3]. FWHM value of the peaks is $\sim 1.8 \mathrm{Da}$ for this ion at $m / z 3200 \mathrm{Da}$, which corresponds to mass resolution of 1800 . Figure $2 \mathrm{c}$ contains the ESI mass spectra of IgG001 deconvoluted in the range from $\mathrm{m} / \mathrm{z} 2900$ to 3500 by using a defined number of iterations, eight. Figure $2 b$ and $c$ show, that the $\mathrm{V}$ and $\mathrm{W}$ modes provide similar resolution of the peaks of glycosylation profile. Deconvolution of the multiply charged ions from the higher $m / z$ range (for example, $m / z$ 3800-4800) produced broader peaks and more tailing, especially for $\mathrm{V}$ mode (data not shown).

Deconvolution is a process of transformation of multiply charged electrospray ions (seen in Figure 2a) into MW value of an analyte using a software algorithm [14-16]. In Figure 2c, the deconvolution was stopped after eight iterations when peak width was approxi- mately equal to the calculated FWHM values (30 to 40 Da) shown in Figure 1. The deconvoluted mass spectra in Figure 2c correlate well with the original ESI mass spectra in Figure 2b, and have the same number of peaks and similar intensity ratios. No major differences in glycosylation profiles were observed in the spectra acquired in $\mathrm{V}$ and $\mathrm{W}$ modes. Figure $2 \mathrm{~d}$ shows the deconvoluted ESI mass spectra for IgG001 deconvoluted to convergence, when the omega value reaches one. After deconvolution to convergence, several extra peaks appeared. We suggest that these extraneous peaks are artifacts of the deconvolution algorithm because they are not visible on the original ESI mass spectra shown in Figure 2b. Although the peaks due to the galactose variants remain approximately the same in Figure 2d, the putative peaks shift in mass and intensity. The putative peaks shift when switching from $\mathrm{V}$ to $\mathrm{W}$ mode and also when width at half height used for deconvolution changes, for example, from 1.4 Da in $\mathrm{V}$ mode to $1.2 \mathrm{Da}$ in $\mathrm{W}$ mode in Figure 2d. Several deconvolution artifacts and their sources have been described in the literature, including (1) synthetic "overtone" corresponding to doubling, tripling, etc. of both the molecular mass and the number of charges, and fractional charges [14]; (2) side peaks closest to the molecular mass peak $M$, which occur at $\left[\left(i_{\max } \pm 1\right) /\right.$ $\left.\mathrm{i}_{\text {max }}\right] M$, where $\mathrm{i}_{\max }$ is the highest number of charges used for deconvolution [14]; and (3) when two or more different species or impurities are present in the sample [15]. It seems that the artificial peaks near the molecular peaks described in this paper do not belong to the above categories. We attribute these artificial peaks to the periodic galactose peaks separated by $162 \mathrm{Da}$. These glycosylation peaks introduce an unexpected (and not completely understood at this time) challenge to the deconvolution algorithm. It should be noted, that the artificial peaks were not observed in the deconvoluted mass spectra of our other protein molecules, which do not possess the heterogeneity caused by sugar moieties as, for example, in cytochrome $c$ in reference [14].

In mass spectrometry, precision improves with the increasing ion count and, therefore, increasing amount of injected sample. We usually inject $\sim 4 \mu \mathrm{g}$ of the protein, to produce the ion intensity above 40 , which is equivalent to $\sim 10^{5}$ of multiply charged IgG ions acquired between $\mathrm{m} / \mathrm{z} 2500$ to 4000 . Injection of higher amounts of protein leads to broadening of the chromatographic peak, carryover, and nonlinear response of UV detector.

Fluctuations of ambient temperature can affect the mass accuracy of TOF instruments by causing elongation of the flight tube with increasing temperature. Variations may also be caused by a high voltage drift during heating of power supplied and by other voltage fluctuations. Many commercial TOF mass spectrometers are equipped with an automatic temperature correction function. For example, $70 \mathrm{ppm} /{ }^{\circ} \mathrm{C}$ was a typical temperature correction coefficient for Waters Q-TOF Micro and $33 \mathrm{ppm} /{ }^{\circ} \mathrm{C}$ for LCT Premier used in this 


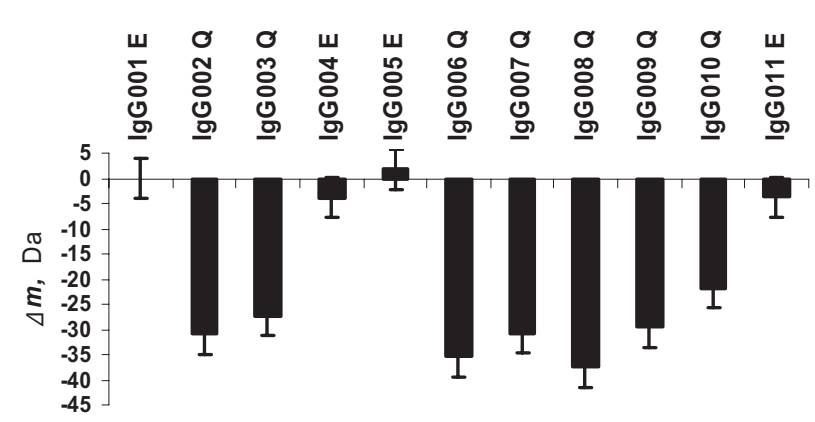

Figure 3. Mass differences $(\Delta m)$ between the measured and calculated MW values of eleven different intact antibodies. The correction was made using the calibration curve for a monoclonal antibody IgG001, which was used as an external standard. Letters $\mathrm{E}$ and $\mathrm{Q}$ next to each antibody indicate the presence of either glutamic acids or glutamine residues at the N-termini of the two heavy chains of intact antibody. The N-terminal glutamine residues are susceptible to conversion to pyroglutamic acids with mass decrease by 17 Da per chain, or total mass decrease of $34 \mathrm{Da}$ for two chains. The light chains of all antibodies have N-terminal glutamic acid and aspartic acid residues, which are stable with respect to the cyclization.

study. When the coefficient was chosen correctly and ambient temperature fluctuations in the room were not large, the precision of mass measurements was around 1.5 Da. It was measured from deconvoluted ESI mass spectra like in Figure 2c. After four consecutive injections of the same antibody, standard deviation of mass measurements of peaks G0F/G0F, G1F/G0F, and G1F/ G1F was equal to 1.5, 1.1, and 3.8, correspondently (data not shown). To compensate for possible mass deviations caused by larger temperature fluctuations and other instrument variations that may occur during long-term operation, we periodically injected external standard (IgG001) antibody to generate a calibration curve, which was utilized to correct the measured masses of unknown antibodies injected in the same series. The long-period mass fluctuations (within days) were within 3 Da.

MW values for the ten monoclonal antibodies were measured according to the described procedure and plotted as a deviation from the calculated MW values in Figure 3. The measured MW values of four antibodies (IgG001, IgG004, IgG005, and IgG011), which possessed only N-terminal glutamic acid residues (E), were within $4 \mathrm{Da}$ from their calculated MW values, including $-4 \mathrm{Da}$ for IgG004, +2 Da for IgG005, and -4 Da for IgG011. Presuming that these four antibodies do not have any other modifications, mass accuracy was within 4 Da or $30 \mathrm{ppm}$. This value ( $\pm 4 \mathrm{Da})$ was used as the error bar in Figure 3. The source of this error can be attributed to different glycosylation profiles and distributions of multiply changed ions of the different antibodies. Note that the 30-ppm mass accuracy determined for different antibodies is different from the 10-ppm precision measured by multiple injections of the same antibody. The other seven antibodies, each containing two $\mathrm{N}$-terminal glutamine residues $(Q)$ on heavy chains, showed decreases in MW values close to $34 \mathrm{Da}$. N-terminal pyro- glutamic acid formation, which leads to a loss of -17 $\mathrm{Da}$ on one heavy chain and hence a total mass shift of $-34 \mathrm{Da}$ on the intact molecule including two heavy chains, was identified as the source of the mass reduction. The percentage of N-terminal pyroglutamate was estimated as following. For example, the corrected MW value of the antibody IgG009 was 30 Da below the calculated MW value. The -30 Da mass difference observed for IgG009 corresponds to a mixture of $88 \%$ pyroglutamate and $12 \%$ glutamate (Table 2 ). The largest percentage of the remaining $\mathrm{N}$-terminal glutamine of $36 \%$ was measured on antibody IgG010 (Figure 3 and Table 2). The relative abundance of the N-terminal glutamine and pyroglutamic acid residues was confirmed by other analytical methods [17].

\section{Conclusions}

LC/MS analysis of intact proteins using ESI orthogonal TOF mass spectrometry is a useful tool for the analysis of monoclonal antibodies. ESI-TOF MS allows for the characterization of various sugar forms that are typically found in antibodies. The best mass resolution was achieved for ions with higher charge states (lower $\mathrm{m} / \mathrm{z}$ value). Peak tailing and broadening was observed for the lower changed states, especially in V mode, probably due to the solvents adducts of TFA. After deconvolution to complete convergence, artificial peaks appeared among the periodic glycosylation peaks. They were eliminated by interrupting the deconvolution after a limited number of iterations (typically eight). For LC/MS analysis of intact antibodies, precision of mass measurements after four consecutive injections of the same antibody was $10 \mathrm{ppm}( \pm 1.5 \mathrm{Da})$, and mass accuracy in measuring different antibodies was $30 \mathrm{ppm}( \pm 4$ Da). These values were achieved by calibrating mass spectrometer with a standard antibody of similar mass and in the same $\mathrm{m} / \mathrm{z}$ range, by periodically injecting a standard antibody to correct for possible long-term mass fluctuations, and by injecting $4 \mu \mathrm{g}$ of protein for high signal intensity. The improved mass accuracy allowed analysis of modifications such as N-terminal pyroglutamic acid formation $(+17 \mathrm{Da})$ on intact antibody despite the fact that this modification cannot be

Table 2. The percentage of N-terminal pyroglutamic acid derived from the mass difference between the measured and calculated MW values $(\Delta \mathrm{m})$ in Figure 3

\begin{tabular}{cc}
\hline & \% pyroglu \\
\hline \hline $\lg G 002$ & 91 \\
$\lg G 004$ & 80 \\
$\lg G 007$ & 104 \\
$\operatorname{lgG} 008$ & 88 \\
$\operatorname{lgG} 009$ & 110 \\
$\operatorname{lgG010}$ & 87 \\
$\lg 0011$ & 64 \\
\hline
\end{tabular}

An error of $\pm 12 \%$ should be used based on mass accuracy of intact antibody of $\pm 4 \mathrm{Da}$. 
resolved by MS. The N-terminal glutamine residues of recombinant monoclonal IgG antibodies are almost completely converted to pyroglutamic acid during production process.

\section{Acknowledgments}

The authors thank Stephen Shriver of Water Corporation for optimization of instrument parameters. They also acknowledge Douglas Rehder for his important contributions in the developing the HPLC method, Dr. Gerd Kleemann for his critical review of the manuscript. They thank Dr. Roman Zubarev and Dr. Alexander Makarov for fruitful discussions and Dr. Michael Senko for his help with IsoPro.

\section{References}

1. Brekke, O. H.; Sandlie, I. Therapeutic antibodies for human diseases at the dawn of the twenty-first century. Nat. Rev. Drug Discov. 2003, 2, 52-62.

2. Wright, A.; Morrison, S. L. Effect of glycosylation on antibody function: Implications for genetic engineering. Trends Biotechnol. 1997, 15, 26-32.

3. Krapp, S.; Mimura, Y.; Jefferis, R.; Huber, R.; Sondermann, P. Structural analysis of human IgG-Fc glycoforms reveals a correlation between glycosylation and structural integrity. J. Mol. Biol. 2003, 325, 979-989.

4. Harris, R. J. Processing of C-terminal lysine and arginine residues of proteins isolated from mammalian cell culture. J. Chromatogr. A 1995, $705,129-134$

5. Jones, G. H. Cell-free synthesis of amino-terminal L-pyroglutamic acid. Biochemistry 1974, 13, 855-860.
6. Abraham, G. N.; Podell, D. N. Pyroglutamic acid. Nonmetabolic formation, function in proteins and peptides, and characteristics of the enzymes effecting its removal. Mol. Cell Biochem. 1981, 38, 181-190.

7. Zhang, Z.; Henzel, W. J. Signal peptide prediction based on analysis of experimentally verified cleavage sites. Protein Sci. 2004, 13, 2819-2824.

8. Kabat, E. A.; Wu, T. T.; Perry, H. M.; Gottesman, K. S.; Foeller, S. Sequences of proteins of immunological interest, 5th ed.; NIH Publication: 1991, pp 91-3242.

9. Kaplan, A. P.; Hood, L. E.; Terry, W. D.; Metzger, H. Amino terminal sequences of human immunoglobulin heavy chains. Immunochemistry 1971, 8, 801-811.

10. Beck, A.; Bussat, M. C.; Klinguer-Hamour, C.; Goetsch, L.; Aubry, J. P.; Champion, T.; Julien, E.; Haeuw, J. F.; Bonnefoy, J. Y.; Corvaia, N. Stability and CTL activity of N-terminal glutamic acid containing peptides. J. Peptide Res. 2001, 57, 528-538.

11. Shih, F. F. Analysis of glutamine, glutamic acid and pyroglutamic acid in protein hydrolysates by high-performance liquid chromatography. J. Chromatogr. 1985, 322, 248-256.

12. Bongers, J.; Cummings, J. J.; Ebert, M. B.; Federici, M. M.; Gledhill, L.; Gulati, D.; Hilliard, G. M.; Jones, B. H.; Lee, K. R.; Mozdzanowski, J.; Naimoli, M.; Burman, S. Validation of a peptide mapping method for a therapeutic monoclonal antibody: What could we possibly learn about a method we have run 100 times? J. Pharm. Biomed. Anal. 2000, 21, 1099-1128.

13. Dillon, T. M.; Bondarenko, P. V.; Rehder, D. S.; Pipes, G. D.; Kleemann, G. R.; Ricci, M. S. Optimization of a reversed-phase LC/MS method for characterizing recombinant antibody heterogeneity and stability. J. Chromatogr. A 2006, in press.

14. Mann, M.; Meng, C. K.; Fenn, J. B. Interpreting mass spectra of multiply charged ions. Anal. Chem. 1989, 61, 1702-1708.

15. Reinhold, B. B.; Reinhold, V. N. Electrospray ionization mass spectrometry: Deconvolution by an entropy-based algorithm. J. Am. Soc. Mass Spectrom. 1992, 3, 207-215.

16. Ferrige, A. G.; Seddon, M. J.; Green, B. N.; Jarvis, S. A.; Skilling, J. Disentangling electrospray spectra with maximum entropy. Rapid Commun. Mass Spectrom. 1992, 6, 707-711.

17. Rehder, D. S.; Dillon, T. M.; Pipes, G. D.; Bondarenko, P. V. Reversedphase LC/MS analysis of reduced monoclonal antibodies in pharmaceutics. J. Chromatogr. A 2006, 1102, 164-175. 